\section{Hypertensive brainstem encephalopathy involving deep supratentorial regions: does only blood pressure matter?}

\author{
Jong-Ho Park, ${ }^{1}$ Sung-Min Kim, ${ }^{2}$ \\ Hyung-Woo Shin, ${ }^{1}$ Sang Joon An ${ }^{1}$ \\ 'Department of Neurology, Myongji \\ Hospital, Kwandong University College of \\ Medicine, Goyang, Republic of Korea; \\ 2Department of Neurology, Seoul \\ National University Hospital, Seoul, \\ Republic of Korea
}

\begin{abstract}
We report on a 42-year-old female patient who presented with high arterial blood pressure of 245/150 mmHg and hypertensive brainstem encephalopathy that involved the brainstem and extensive supratentorial deep gray and white matter. The lesions were nearly completely resolved several days after stabilization of the arterial blood pressure. Normal diffusionweighted imaging findings and high apparent diffusion coefficient values suggested that the main pathomechanism was vasogenic edema owing to severe hypertension. On the basis of a literature review, the absolute value of blood pressure or whether the patient can control his/her blood pressure seems not to be associated with the degree of the lesions evident on magnetic resonance imaging. It remains to be determined if the acceleration rate and the duration of elevated arterial blood pressure might play a key role in the development of the hypertensive encephalopathy pattern.
\end{abstract}

\section{Introduction}

Hypertensive encephalopathy (HE) is a lifethreatening condition characterized by severe hypertension, altered mental status, headache, dizziness, visual changes, and seizure. The condition is reversible if promptly diagnosed and treated, but if treatment is delayed it may progress to coma or death. ${ }^{1,2}$ Because the lesions improve after the stabilization of paroxysmally accelerated hypertension, HE is classified as a reversible posterior leukoencephalopathy syndrome. The most common abnormality detected by magnetic resonance imaging (MRI) is hyperintensity of T2-weighted and fluid-attenuated inversion recovery (FLAIR) images of the white matter bilaterally, especially in the parieto-occipital areas. This is consistent with cerebral edema.
Predominant hypertensive brainstem encephalopathy (HBE) with supratentorial involvement is another form of HE. ${ }^{3-18}$ Cases of HBE involving supratentorial deep gray and white matter extensively are rare. ${ }^{17,18} \mathrm{We}$ describe here a patient with HBE involving deep supratentorial gray and white matter. In addition, we have tried to elucidate the possible pathophysiological mechanism involved, on the basis of a literature review.

\section{Case Report}

A 42-year-old woman (high-school graduate) had been diagnosed with hypertension two years earlier. Following diagnosis, she was prescribed antihypertensive medication for one month only. Her mother and her elder and younger sisters also had hypertension.

One day before her admission, she developed dizziness. When she was first examined on admission day, she showed drowsiness and mental confusion. She also complained of an inability to stand or to walk owing to dizziness. Her vital signs included a blood pressure of $245 / 150 \mathrm{~mm} \mathrm{Hg}$, a pulse rate of $94 \mathrm{~min}$, a respiration rate of $18 / \mathrm{min}$, and a body temperature of $36.5^{\circ} \mathrm{C}$. There was no abnormal finding on physical examination. On neurological examination, her mental status was drowsy. She had marked truncal and limb ataxia. She did not present with dysarthria, facial or limb weakness, or sensory loss. Cranial nerve examination, including extraocular movement and bulbar function, was normal. Her deep tendon reflexes were normal and without pathological reflex.

On the second day after admission (46 hours after symptom onset), a brain MRI examination was conducted. Extensive hyperintense changes in the periventricular and subcortical deep white matter, the internal capsule, the thalamus, the midbrain, the pons, and the cerebellum were revealed bilaterally on fluid-attenuated inversion recovery (FLAIR) images (repetition time/echo time (TR/TE) $11000 / 140 \mathrm{~ms}$ ), on T2-weighted images (TR/TE $4230 / 100 \mathrm{~ms}$ ), as well as on apparent diffusion coefficient (ADC) mapping (Figure 1). The same lesions showed near isointensity on diffusion-weighted (DWI, b-values $=1000 \mathrm{~s} / \mathrm{mm}^{2}$ ) and T1-weighted (TR/TE 474/12 ms) images. These findings were suggestive of widespread vasogenic edema. A lacunar infarction (asymptomatic old lesion) was noted incidentally in the right anterior thalamus. No evidence of abnormal enhancement was found in the brain parenchyma. MR angiography revealed no abnormalities in the major intra- or extracranial arteries. Both posterior cerebral arteries were raised from the vertebrobasilar circulation with no fetal supply.
Correspondence: Jong-Ho Park, Department of Neurology, Myongji Hospital, Kwandong University College of Medicine, 697-24 Hwajeong-dong, Deokyang-gu Goyang-si, Gyeonggi-do 412-270, Republic of Korea.

E-mail: neurocraft@kd.ac.kr

Key words: hypertensive encephalopathy, vasogenic edema, hypertension, acceleration, duration, MR imaging.

Received for publication: 30 0ctober 2009.

Revision received: 26 March 2010.

Accepted for publication: 2 April 2010.

This work is licensed under a Creative Commons Attribution 3.0 License (by-nc 3.0).

(C) Copyright J-H Park et al., 2010

Licensee PAGEPress, Italy

Neurology International 2010; 2:e9

doi:10.4081/ni.2010.e9

Serum laboratory findings including glucose, electrolyte, arterial blood gas analysis, hepatic enzyme, and thyroid hormone (T3, T4, thyroid-stimulating hormone) levels were within normal limits except for mildly elevated urea nitrogen $(31.1 \mathrm{mg} / \mathrm{dL}$, normal: 5.0-25.0 $\mathrm{mg} / \mathrm{dL})$ and creatinine $(1.5 \mathrm{mg} / \mathrm{dL}$, normal: 0.5 $1.4 \mathrm{mg} / \mathrm{dL})$. An enzyme study for differentiation from Fabry's disease revealed that the alpha-galactosidase A activity was $92 \%$ (normal: >85\%). A cerebrospinal fluid study showed unremarkable findings: normal opening pressure ( $\left.130 \mathrm{~mm} \mathrm{H}_{2} \mathrm{O}\right)$; white blood cell count, $2 / \mathrm{mm}^{3}$; a glucose level, $86 \mathrm{mg} / \mathrm{dL}$ (normal: $40-80 \mathrm{mg} / \mathrm{dL}$ ); and protein concentration, $49 \mathrm{mg} / \mathrm{dL}$ (normal: 12-60 mg/dL). An electroencephalogram showed diffuse theta to delta slowing. An electrocardiogram revealed left ventricular hypertrophy, and thoracic echocardiography showed severe concentric left ventricular hypertrophy with diastolic dysfunction. Abdominal and pelvic computed tomography (CT) scans showed no evidence of pheochromocytoma, but right renal artery stenosis was detected (40\%).

We immediately started intravenous infusion of a calcium-channel blocker (nicardipine hydrochloride, $50 \mathrm{mg}$ ), which lowered her systolic pressure to $140-170 \mathrm{~mm} \mathrm{Hg}$ and her diastolic pressure to $70-90 \mathrm{~mm} \mathrm{Hg}$. This was followed by an oral calcium-channel blocker (amlodipine, $10 \mathrm{mg}$ ), an angiotensin-converting enzyme inhibitor (perindopril, $8 \mathrm{mg}$ ), an angiotensin II receptor blocker (valsartan, 160 $\mathrm{mg}$ ), a beta blocker (bisoprolol, $2.5 \mathrm{mg}$ ) and diuretics (torasemide, $5 \mathrm{mg}$ and spironolactone, $25 \mathrm{mg}$ ). After the elevated blood pressure was consistently lowered for a week, she regained consciousness gradually over a period of three days. On the fourth day following admission, she scored 10/30 on the Korean 
mini-mental status examination (K-MMSE) $;^{19}$ furthermore, her clinical dementia rating (CDR) and global deterioration scale (GDS) score were 3 and 6 , respectively. On the seventh day, she could move independently without ataxia and her score on the follow-up KMMSE was 23/30. She was discharged on the $23^{\text {rd }}$ day post-admission and subsequently was followed up and prescribed antihypertensive medication. During follow-up and eight months after admission, her score on the $\mathrm{K}$ MMSE, CDR, and GDS were 20/30, 0.5, and 4, respectively. Her follow-up MRI revealed that the abnormal hyperintense signals had diminished markedly on the FLAIR images, except for some leukoaraiosis (Figure 2).

\section{Discussion}

We present a female patient with untreated hypertension who developed HBE involving the entire cerebellum and the deep supratentorial regions. On admission, she presented with drowsiness, vertigo, and ataxia without accompanying motor weakness or visual disturbances. Most cases with brainstem or cerebellar involvement show clinicoradiologic dissociation. ${ }^{20}$ However, our patient demonstrated lesions so extensive as to show dizziness as well as limb and truncal ataxia. These symptoms might result from cerebellar lesions, and the drowsiness probably was secondary to involvement of the ascending reticular activating system in the brainstem. The patient was diagnosed with HE based on the following three criteria: i) severe hypertension, ii) diffuse symmetrical MRI lesions with no compatible stroke lesions in DWI, iii) subsequent improvement of clinical symptoms and radiological findings after the normalization of blood pressure.

The differential diagnosis included cerebral thromboembolism, encephalitis, hypoxia, osmotic demyelination syndromes, or metabolic encephalopathy. The affected territory and symmetrical lesions described here were not correlated with simple thromboembolic stroke because of the lack of major brainstem signs, the absence of acute ischemic findings on initial DWI, and the reversible radiological findings with a clinical recovery. In consideration of the clinicoradiologic aspects of the case and the normal CSF findings, the possibility of an inflammatory disease (e.g. acute disseminated encephalomyelitis) was very unlikely because of the absence of a preceding infection or of vaccination. Symmetrical signal changes in the brain MRI are observed in patients with hypoxia, osmotic demyelination syndromes (central pontine and extrapontine myelinolysis), or metabolic disorders such as hyperglycemia, liver cirrhosis, and Fabry's disease.

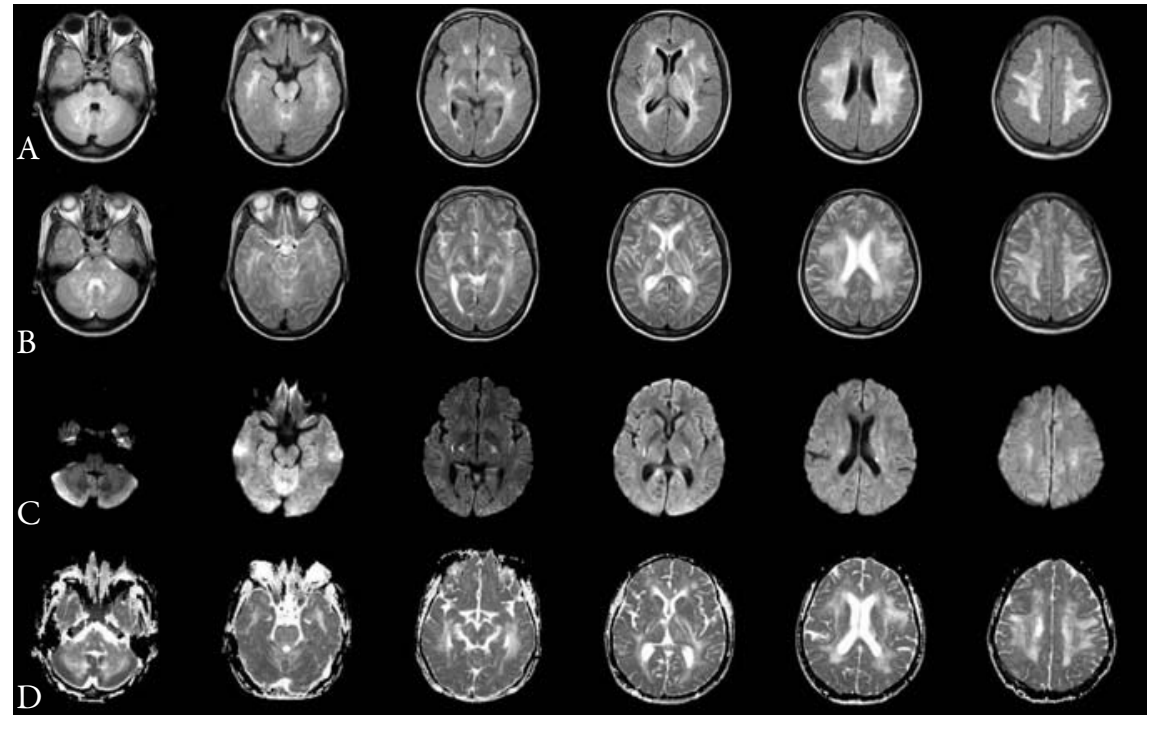

Figure 1. Brain magnetic resonance imaging 46 hours after symptom onset showing hyperintensity involving the periventricular and subcortical deep white matter, the internal capsule, the thalamus, the midbrain, the pons, and the cerebellum bilaterally, on fluid-attenuated inversion recovery (FLAIR) images (A), on T2-weighted images (B), and on apparent diffusion coefficient (ADC) mapping (D). The same lesions showed near isointensity on diffusion-weighted imaging (DWI) (C).

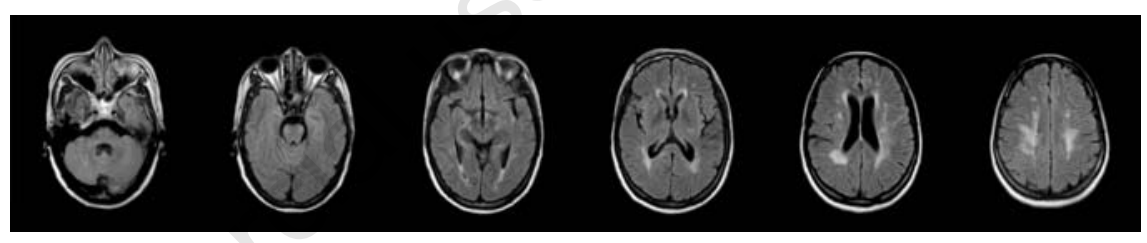

Figure 2. Follow-up magnetic resonance imaging after eight months, showing significant diminution of hyperintensity on the FLAIR sequence, except for some leukoaraiosis. The reversible changes of clinical symptoms and radiological findings after the normalization of blood pressure are indicative of hypertensive encephalopathy.

However, there was no history of hypoxic brain damage, hyperglycemia, liver disease, or electrolyte abnormality in our patient. The resolution of the MRI findings helped to eliminate the possibility of the above diseases. Furthermore, the possibility of Fabry's disease was excluded because the serum enzyme assay (galactosidase A) was normal.

Our patient had extensive high-intensity signals in the brainstem and cerebellum, as well as in the subcortical deep white and gray matter in T2-weighted and FLAIR images. However, they were isointense on DWI whereas hyperintensity was seen in the initial ADC mapping. The follow-up FLAIR imaging showed a near-reversible change following control of blood pressure. These findings revealed that the lesions were predominantly a result of vasogenic edema, which is a hallmark of HE. The accepted mechanism of HE is vasogenic edema that results from blood-brain barrier disruption and extravasation of fluid and protein. This is caused by a rapidly elevated blood pressure that exceeds the autoregulatory limit of the brain vasculature. ${ }^{21,22}$ The hypothesis that the essential mechanism of HE is severe hypertension-induced vasogenic edema is supported by a brain SPECT (single-photon emission-computed tomography) study. This study showed increased perfusion in the vicinity of the brain, and the abnormal MRI signal showed almost complete resolution after the normalization of blood pressure., ${ }^{423}$

Although the typical MRI abnormality observed in cases of HE is hyperintensity in the white matter in the parieto-occipital areas bilaterally, many cases of HBE have also been reported. Table 1 shows the reported cases of HE to date and the variable patterns of HBE according to the severity of the lesion: i) posterior dominant supratentorial lesion (HE) ${ }^{24}$ ii) HBE only, ${ }^{3-10}$ iii) predominant HBE with relative sparing of the supratentorial regions, ${ }^{11-16}$ and iv) HBE extending into the whole deep supratentorial regions..$^{17,18}$ The latter two cases $^{17,18}$ are similar to our case.

It is unclear how the different pathophysiological mechanisms could be explained between patients who have HBE with supratentorial lesions, including the posterior corti- 
cal region, and those who do not. Mild acceleration of hypertension-produced edema occurred mainly in the supratentorial white matter. ${ }^{18}$ Severe acceleration of hypertension ended in more extensive supratentorial edema and extension into the infratentorial regions. ${ }^{18}$ Table 1 illustrates that the initial blood pressure in patients with HBE usually was higher than in those with typical HE. In three patients with $\mathrm{HBE}$, however, the absolute value of blood pressure or whether or not the patient controls his/her blood pressure seems not to be associated with the degree of the MRI lesions.

The upper limit of the autoregulatory plateau of cerebral blood flow in the deep regions (e.g. thalamus, basal ganglia, or brainstem) is higher than that in the cerebral cortex in normotensive and spontaneously hypertensive rat models. ${ }^{25}$ The deep regions of the brain are supplied directly from branches of the parent artery (e.g. the middle or posterior cerebral artery or basilar arteries), while the cortex and subcortex are fed by the terminal pial arteries. ${ }^{25}$ Therefore, it is likely that more intense arterial blood pressure caused vasogenic edema in the deep regions. Regarding the hyperperfusion surges caused by hypertension, although the anterior circulation is known to have more significant sympathetic innervation than does the posterior circulation ${ }^{26}$ we can also suggest that the HE pattern might depend on anatomical characteristics and the severity of hypertension (acceleration rate and duration). The major arteries from the carotid artery comprise at least two paired systems (e.g. the anterior and middle cerebral arteries) and have a larger diameter than does the basilar artery; the latter is involved in a single circulation and the diameter of each vertebral artery is smaller than that of the internal carotid arteries. Mild acceleration of hypertension might affect small-caliber arteries (distal pial branches), such as in the parieto-occipital area as in a report of the influence of mild acceleration of hypertension on the supratentorial white matter. ${ }^{18}$ Paroxysmal and lasting acceleration of hypertension, on the other hand, would have its first effect on the brainstem.

Apart from the extensive deep white and gray matter involvement, supratentorial cortices and subcortices were relatively uninvolved in our patient. It is plausible that early extensive fluid leakage from the proximal arteries might spare the supratentorial superficial regions (end portions), including the parieto-occipital regions. It remains to be determined whether the acceleration rate and the duration of elevated arterial blood pressure might have a key role in the development of the HE pattern.

Table 1. Clinical features of patients with hypertensive encephalopathy and those with variable patterns of hypertensive brainstem encephalopathy who underwent brain computerized tomography scanning, magnetic resonance imaging, or both.

\begin{tabular}{|c|c|c|c|}
\hline Author & Sex/Age & Past medical Hx & SBP/DBP, mm Hg \\
\hline $\begin{array}{l}\text { Posterior domir } \\
\text { Hauser }^{24}\end{array}$ & $\begin{array}{c}\text { ratentorial } \\
\mathrm{M} / 4.5 \\
\mathrm{~F} / 34 \\
\mathrm{~F} / 53\end{array}$ & $\begin{array}{c}\text { None } \\
\text { None } \\
\text { uHT, CRF }\end{array}$ & $\begin{array}{l}236 / 172 \\
200 / 140 \\
220 / 140\end{array}$ \\
\hline $\begin{array}{l}\text { Only HBE } \\
\text { Karasawa }^{3} \\
\text { Ono }^{4} \\
\text { Kanazawa }^{5} \\
\text { Gamanagatti }^{6} \\
\text { Uchino }^{7} \\
\text { Kang }^{8} \\
\text { Shintani } \\
\\
\text { Bhagavati }^{10}\end{array}$ & $\begin{array}{l}M / 63 \\
F / 67 \\
M / 75 \\
M / 76 \\
M / 60 \\
M / 29 \\
M / 42 \\
M / 53 \\
F / 45 \\
M / 85 \\
M / 46 \\
M / 42 \\
\end{array}$ & $\begin{array}{c}\text { cHT, DM } \\
\text { DM } \\
\text { None } \\
\text { cHT } \\
\text { None } \\
\text { None } \\
\text { uHT } \\
\text { uHT } \\
\text { uHT, CRF } \\
\text { None } \\
\text { uHT, CRF } \\
\text { cHT }\end{array}$ & $\begin{array}{l}240 / 80 \\
204 / 106 \\
200 / 120 \\
230 / 100 \\
220 / 150 \\
240 / 160 \\
240 / 160 \\
170 / 100 \\
190 / 130 \\
221 / 112 \\
244 / 150 \\
238 / 147\end{array}$ \\
\hline $\begin{array}{l}\text { Predominant Hl } \\
\text { Chang }^{11} \\
\text { de Seze }^{12} \\
\text { Yasuda }^{13} \\
\text { Nagata }^{14} \\
\text { Fujiwara }^{15} \\
\text { Doil }^{16}\end{array}$ & $\begin{array}{c}\text { relative sp } \\
\mathrm{F} / 54 \\
\mathrm{M} / 49 \\
\mathrm{M} / 41 \\
\mathrm{~F} / 52 \\
\mathrm{M} / 45 \\
\mathrm{M} / 67 \\
\mathrm{M} / 38 \\
\mathrm{M} / 35 \\
\mathrm{~F} / 52\end{array}$ & $\begin{array}{c}\text { tentorial regions } \\
\text { uHT } \\
\text { cHT } \\
\text { cHT } \\
\text { uHT } \\
\text { uHT } \\
\text { uHT, DM, CRF } \\
\text { uHT } \\
\text { CRF } \\
\text { uHT }\end{array}$ & $\begin{array}{l}210 / 144 \\
211 / 156 \\
220 / 120 \\
220 / 150 \\
250 / 160 \\
230 / 122 \\
240 / 180 \\
180 / 118 \\
200 / 130\end{array}$ \\
\hline $\begin{array}{l}\text { HBE plus exten } \\
\text { Yoshida }^{17} \\
\text { Kumai }^{18}\end{array}$ & $\begin{array}{c}\text { ratentorial } \\
\mathrm{M} / 58 \\
\mathrm{M} / 73\end{array}$ & $\begin{array}{c}\text { uHT, CRF } \\
\text { uHT }\end{array}$ & $\begin{array}{l}210 / 90 \\
300 / 160\end{array}$ \\
\hline
\end{tabular}

HE, hypertensive encephalopathy; HBE, hypertensive brainstem encephalopathy; SBP, systolic blood pressure; DBP, diastolic blood pressure; M, male; F, female; uHT, uncontrolled or poorly controlled hypertension; cHT, controlled or chronic hypertension; DM, diabetes mellitus; CRF, chronic renal failure.

\section{References}

1. Venkata C, Ram S. Hypertensive encephalopathy: recognition and management. Arch Intern Med 1978;138:1851-3.

2. Gifford RW. Management of hypertensive crises. JAMA 1991;266:829-35.

3. Karasawa S, Kawanami T, Kimura H, et al. An unusual case of hypertensive encephalopathy involving the brain stem. Intern Med 2004;43:448-9.

4. Ono Y, Manabe Y, Hamakawa Y, et al. Localized lesions on MRI in a case of hypertensive brainstem encephalopathy. Intern Med 2005;44:1002-5.

5. Kanazawa M, Sanpei K, Kasuga K. Recurrent hypertensive brainstem encephalopathy. J Neurol Neurosurg Psychiatry 2005;76:888-90.

6. Gamanagatti S, Subramanian S. Hypertensive encephalopathy: isolated pons involvement mimicking central pontine myelinolysis. Korean J Radiol 2006;7:2189
7. Uchino M, Haga D, Nomoto $\mathrm{J}$, et al. Brainstem involvement in hypertensive encephalopathy: A report of two cases and literature review. Eur Neurol 2007;57:2236.

8. Kang SY, Choi JC, Kang JH. Two cases of hypertensive encephalopathy involving the brainstem. J ClinNeurol 2007;3:50-2.

9. Shintani S, Hino T, Ishihara $S$, et al. Reversible brainstem hypertensive en cephalopathy (RBHE): Clinicoradiologic dissociation. Clin Neurol Neurosurg 2008;110:1047-53

10. Bhagavati S, Chum F, Choi J. Hypertensive encephalopathy presenting with isolated brain stem and cerebellar edema. J Neuroimaging 2008;18:454-6.

11. Chang GY, Keane JR. Hypertensive brainstem encephalopathy: three cases presenting with severe brainstem edema. Neurology 1999;53:652-4.

12. de Seze J, Mastain B, Stojkovic T, et al. Unusual MR findings of the brain stem in arterial hypertension. Am J Neuroradiol 2000;21:391-4. 
13. Yasuda Y, Akiguchi I, Imai T, et al. Hypertensive brainstem encephalopathy. Intern Med 2003;42:1131-4.

14. Nagata M, Maeda M, Tsukahara H, et al. Brainstem hypertensive encephalopathy evaluated by line scan diffusion-weighted imaging. Am J Neuroradiol 2004;25:803-6.

15. Fujiwara H, Momoshima S, Kuribayashi S, et al. Hypertensive encephalopathy of brain stem with minimal supratentorial involvement: A rare manifestation of hypertensive encephalopathy. Radiat Med 2005;23:504-7.

16. Doi Y, Kimura F, Fujiyama T, et al. Hypertensive brainstem encephalopathy without parieto-occipital lesion -Two case reports. Neurol Med Chir (Tokyo) 2006;46: 75-9.

17. Yoshida K, Yamamoto T, Mori K, et al. Reversible posterior leukoencephalopathy syndrome in a patient with hypertensive encephalopathy. Neurol Med Chir 2001;41:
364-9.

18. Kumai Y, Toyoda K, Fujii K, et al. Hypertensive encephalopathy extending into the whole brainstem and deep structures. Hypertens Res 2002;25:797-800.

19. Kang YW, Na DL, Han SH. A Validity study on the Korean Mini-Mental State Examination (K-MMSE) in dementia patients. J Korean Neurol Assoc 1997;15:300-8.

20. Cruz-Flores S, de Assis Aquino Gondim F, Leira EC. Brainstem involvement in hypertensive encephalopathy: clinical and radiological findings. Neurology 2004;62:14179.

21. Skinhoj E, Strandgaard S. Pathogenesis of hypertensive encephalopathy. Lancet 1973;1:461-2.

22. Weingarten K, Barbut D, Filippi C, et al. Acute hypertensive encephalopathy: findings on spin-echo and gradient-echo MR imaging. Am J Roentgenol 1994;162:665-
70.

23. Schwartz RB, Jones KM, Kalina P, et al. Hypertensive encephalopathy: findings on CT, MR imaging, and SPECT imaging in 14 cases. Am J Roentgenol 1992;159:379-83.

24. Hauser RA, Lacey DM, Knight MR. Hypertensive encephalopathy. Magnetic resonance imaging demonstration of reversible cortical and white matter lesions. Arch Neurol 1988;45:1078-83.

25. Sadoshima S, Fujii K, Yao H, et al. Regional cerebral blood flow autoregulation in normotensive and spontaneously hypertensive rats - effects of sympathetic denervation. Stroke 1986;17:981-4.

26. Beausang-Linder M, Bill A. Cerebral circulation in acute arterial hypertension: protective effects of sympathetic nervous activity. Acta Physiol Scand 1981;111:1939. 